\title{
ZEROS OF A FUNCTION AND OF ITS DERIVATIVE*
}

BY W. J. TRJITZINSKY

Macdonald has proved the following theorem. $\dagger$ Let $f(z)=u(x, y)+i v(x, y)$ be a function of $z$ analytic throughout the interior of a single closed curve $C$, defined by the equation $|f(z)|=M$, where $M$ is a constant. Let $C$ be an ordinary curve in the sense that if $\psi$ be the angle which the tangent to $C$ makes with the $x$-axis, and if the point of contact of the tangent describes the curve $C, \psi$ will increase by $2 \pi$. Then the number of zeros of $f(z)$ in this region exceeds the number of zeros of the derivative $f(z)$ by unity. Our purpose is to generalize this result by showing the conclusion holds true even when $M$ is not a constant, but a function $M=M(x, y)$ analytic and greater than zero within sufficiently large intervals, and $C$ is a curve single, closed, and ordinary (in the sense mentioned above), and satisfying the equation $|f(z)|=M(x, y)$, provided that the point $(x=x(\theta), y=y(\theta))$ describes $C$ once when $\theta$ changes from 0 to $2 \pi$; where $x(\theta)$ and $y(\theta)$ are periodic functions satisfying the equations

$$
\frac{u(x, y)}{M(x, y)}=\cos \theta, \quad \frac{v(x, y)}{M(x, y)}=\sin \theta .
$$

The solvability of these equations implies that the Jacobian of the left members does not vanish identically.

On $C$, let $f(z)=M(x, y) e^{i \theta}$. Then there results a pair of equations (1) whose solutions are $x_{i}=x_{i}(\theta)$ and $y_{i}=y_{i}(\theta)$ of period $2 \pi$ in $\theta$. By hypothesis, among them there is at least one solution, say $x=x(\theta), y=y(\theta)$, representing the curve $C$, and the point $(x=x(\theta), y=y(\theta))$ describes $C$ once as $\theta$ varies from 0 to $2 \pi$.

* Presented to the Society, San Francisco Section, June 18, 1927.

$\dagger$ Proceedings of the London Society, vol. 29 (1898), pp. 576-577; Proceedings of the London Society, (2), vol. 15 (1916), pp. 227-242. 
Letting $M(x(\theta), y(\theta))=N(\theta)$, we observe that $N(\theta)$ as well as $N^{\prime}(\theta)$ is of period $2 \pi$, and for all $\theta, N(\theta)>0$, so that when $z$ describes $C$ and $\theta$ varies from 0 to $2 \pi$, the complex quantity $N^{\prime}(\theta)+i N(\theta)$ describes a closed curve entirely above the real axis; its modulus will return to the initial value and the variation of its argument will be zero. Now on $C$ we have

$$
\begin{aligned}
f(z)= & N(\theta) \cdot e^{i \theta} \\
f^{\prime}(z)= & e^{i \theta} \cdot \frac{d \theta}{d z} \cdot\left[N^{\prime}(\theta)+i N(\theta)\right] \\
f^{\prime \prime}(z)= & e^{i \theta} \cdot f\left\{\left[N^{\prime}(\theta)+i N(\theta)\right] \cdot\left[\frac{d^{2} \theta}{d z^{2}}+i\left(\frac{d \theta}{d z}\right)^{2}\right]\right. \\
& \left.+\left[N^{\prime \prime}(\theta)+i N^{\prime}(\theta)\right]\left(\frac{d \theta}{d z}\right)^{2}\right\} .
\end{aligned}
$$

The excess $e$ of the number of zeros of $f(z)$ over the number of zeros of $f^{\prime}(z)$ within $C$ is

$$
\begin{gathered}
e=\frac{1}{2 \pi i} \int_{C} \frac{f^{\prime}(z)}{f(z)} d z-\frac{1}{2 \pi i} \int_{C} \frac{f^{\prime \prime}(z)}{f^{\prime}(z)} d z \\
=-\frac{1}{2 \pi i} \int_{C} \frac{\left(\frac{d^{2} \theta}{d z^{2}}\right)}{\left(\frac{d \theta}{d z}\right)} d z-\frac{1}{2 \pi i} \int_{C} \frac{d \theta}{d z}\left[\frac{N^{\prime \prime}(\theta)+N^{\prime}(\theta)}{N^{\prime}(\theta)+i N(\theta)}\right. \\
\left.-\frac{N^{\prime}(\theta)}{N(\theta)}\right] d z .
\end{gathered}
$$

The quantity

$$
-\frac{1}{2 \pi i} \int_{c} \frac{\left(\frac{d^{2} \theta}{d z^{2}}\right)}{\left(\frac{d \theta}{d z}\right)} \cdot d z=1,
$$

as can be seen from Whittaker's* presentation of the theorem cited. Hence we have

* Whittaker and Watson, Modern Analysis, 3d ed., p. 121. 


$$
\begin{aligned}
e & =1-\frac{1}{2 \pi i} \int_{C}\left(\frac{d \theta}{d z}\right) \cdot\left[\frac{N^{\prime \prime}(\theta)+i N^{\prime}(\theta)}{N^{\prime}(\theta)+i N(\theta)}-\frac{N^{\prime}(\theta)}{N(\theta)}\right] d z \\
& =1-\frac{1}{2 \pi i} \int_{0}^{2 \pi}\left[\frac{N^{\prime \prime}(\theta)+i N^{\prime}(\theta)}{N^{\prime}(\theta)+i N(\theta)}-\frac{N^{\prime}(\theta)}{N(\theta)}\right] d \theta
\end{aligned}
$$

since $\theta$ varies from 0 to $2 \pi$ when $z$ describes $C$. Moreover

$$
e=1-\frac{1}{2 \pi i} \cdot\left\{\log \left[N^{\prime}(\theta)+i N(\theta)\right]\right\}_{0}^{2 \pi}+\frac{1}{2 \pi i}[\log N(\theta)]_{0}^{2 \pi} .
$$

We know that $N(\theta)$ is real; hence $[\log N(\theta)]_{0}^{2 \pi}=0$. On the other hand, the variation of the argument of $\left[N^{\prime}(\theta)+i N(\theta)\right]$ as $\theta$ changes from 0 to $2 \pi$ is zero, so that $\log \left[\left(N^{\prime}(\theta)\right.\right.$ $+i N(\theta))]_{0}^{2 \pi}=0$. Hence $e=1$. This proves the theorem.

The University of Texas

\section{LINEAR INEQUALITIES IN GENERAL ANALYSIS*}

\section{BY L. L. DINES}

1. Introduction. In his studies in general analysis, E. H. Moore has developed $\dagger$ a theory of the linear functional equation

$$
\xi+J_{\kappa} \xi=\eta .
$$

Here $\xi$ and $\eta$ denote functions (the latter given, the former to be determined) belonging to a class $\mathfrak{M}$ of real-valued functions on a general range $\mathfrak{B}$. The kernel function $\kappa$ belongs to a class $\Re$ which is well defined in terms of the fundamental class $\mathfrak{M}$. A sufficient foundation for the theory is laid by means of postulates upon the class $\mathfrak{M}$ and the functional operation $J$.

The purpose of the present paper is to consider the linear inequality

$$
\xi+J_{\kappa} \xi>0,
$$

* Presented to the Society, September 8, 1927.

$\dagger$ On the foundations of the theory of linear integral equations, this Bulletin, vol. 18 , pp. 334-362. 